\title{
Development of a Relevant Image Processing Method to Characterize the Distribution of Tissue within a Bone Structure
}

\author{
Duarte Ricardo ${ }^{1}$, Delos Vincent ${ }^{1}$, Ramos António², Teschke Marcus ${ }^{3}$ and Mesnard Michel ${ }^{1 *}$ \\ ${ }^{1}$ Université de Bordeaux, I2M, CNRS UMR 5295, FR-33405 Talence, France \\ 'Universidade de Aveiro, Departmento de Engenharia Mecanica, Aveiro, Portugal \\ ${ }^{3}$ Universitätsklinikum Bonn, Bonn, Deutschland
}

\begin{abstract}
In this study one evaluate the efficiency of a new method to characterize the distribution of bone tissue within a human structure that could be wildly explored in research but especially in medical fields due to its simplicity and efficiency. Two different methods were used to characterize the distribution of tissue in the bone structure. The first was open source software (Image $\mathrm{J}$ ) with a dedicated plug-in and the second was commercial software (Simpleware ScanIP). Using temporomandibular joint DICOM files, one evaluated the largest section of the condyle and the distance between the top of the condyle and the section with the least cancellous bone as a way to verify the accuracy of the method and the way that user's skills influence the results. Finally, we built two 3D models based on the extreme cases considering the bone distribution. Results from the two methods were compared in order to validate the new proposed method. The comparison showed that the proposed method has enough feasibility to perform the bone distribution analysis, since the results, precision and repeatability were successfully confirmed. Furthermore, one states that the principal advantage of this method is the faster evaluation of the bone structures with a very satisfying precision which augurs good perspectives during its use in the medical fields.
\end{abstract}

Keywords: Image processing; Bone tissue geometry; Uncertainties; Repeatability

\section{Introduction}

The imaging techniques available to medicine have increased in the past few years with new, more advanced and accurate methods being used. For example, conventional radiography, computed tomography and magnetic resonance tomography play an important role helping surgeons planning their surgery strategies $[1,2]$. To transform and use the information provided by imaging techniques, several software are available in the market to allow the exploration and characterization of the different bone structures and tissues [3]. One well-established approach using this information is based on 3D model reconstruction, through CT scan images. This technique displays a 3D model and allows a better understanding and evaluation of the structure. In the medical field, these methods can be used to prepare the surgical procedure and especially to choose the best implant for each patient when the joint needs replacing [4-8]. Human joints and bone structures present very complex dispositions and geometries which are difficult to evaluate before surgery but have to be considered according to the small area available in some cases [9]. The temporomandibular joint (TMJ) and the jawbone are the most complex joint and bone structures of the human body. Several muscles and nerves need to be preserved to provide a correct joint functioning. Due to their important role in the patient's life and their complexity, maxillofacial surgeries are very sensitive procedures where surgeons need to deal with a small and restricted volume but at the same time they need to cut bone and place an implant.

At present, there are two main temporomandibular joint implants (TMJ implants) available in the market: the TMJ Concept and the Biomet. In order to ensure correct positioning of these implants in the mandibular condyle and the safety of the procedure there are several characteristics that should be taken into account: condyle size, bone quality, implant size and the positioning of the screws [10]. Condyle size is a parameter that should be evaluated in the pre-surgical consultations. Considering the TMJ implant references, in order to place the mandibular part of the implant, the surgeon needs to cut the condyle and the distance available to put the implant will dictate the best option for each case. Another important variable that should be taken into account during TMJ surgeries is the quantity of cancellous bone inside the jawbone in order to choose the best implant and its fixation.

According to the relevant details about these parameters, it is essential to have maximum information about the joint and the bone to prepare a successful surgical intervention. To obtain this information, a few open source software, such as Image-J, have earned their place in the medical field [11-13]. Image-J is based on Java Script, and is an open-source software allowing continuous evolution supported by user contributions through the upload of new packages and functions according to their needs. This software globalization represents a high value advantage when compared with other commercial software that is restricted by their copyrights. The flexibility of this software allowed us to create a new plug-in to characterize the bone tissue and thus provide an accurate but also a faster tool to evaluate the tissue geometry, guiding the surgeons in their decisions through the surgical process, and thus minimizing or avoiding any risk to the patient.

Under these conditions, two characteristics of the human condyle were measured to evaluate the accuracy of the tool and validate the study procedure. These were the area of the largest section of the ramus and the distance between the top point of the condyle and the minimum cancellous bone section inside the mandible.

*Corresponding author: Mesnard Michel, Université de Bordeaux, 12M CNRS UMR 5295, FR-33405 Talence, France, Tel: 33540008952; E-mail: michel.mesnard@u-bordeaux.fr

Received April 30, 2015; Accepted May 22, 2015; Published May 25, 2015

Citation: Ricardo D, Vincent D, António R, Marcus T, Michel M (2015) Development of a Relevant Image Processing Method to Characterize the Distribution of Tissue within a Bone Structure. J Comput Sci Syst Biol 8: 199-202. doi:10.4172/ jcsb.1000189

Copyright: ( 2015 Ricardo D, et al. This is an open-access article distributed under the terms of the Creative Commons Attribution License, which permits unrestricted use, distribution, and reproduction in any medium, provided the original author and source are credited. 


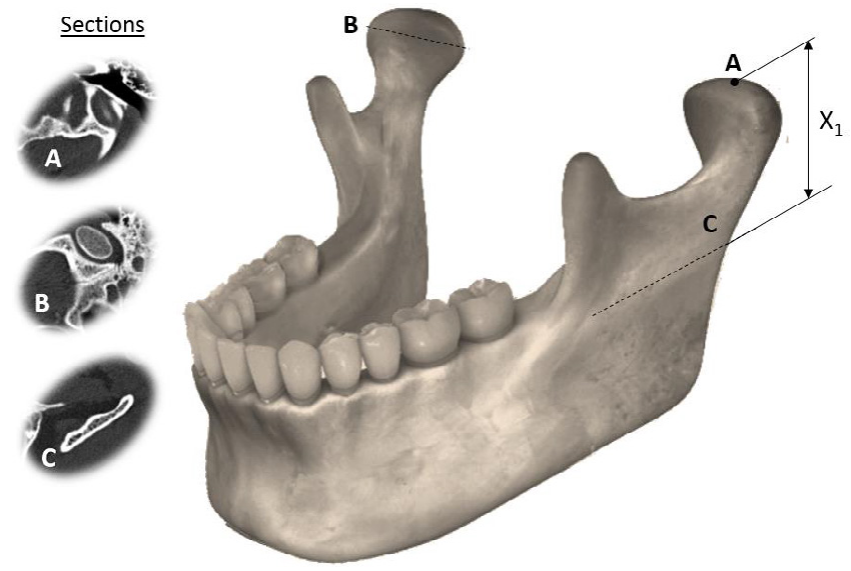

Figure 1: Analysed sections (A: condyle top point; $B$ : condyle largest section $C$ : condyle smallest cancellous bone section; $X_{1}$ : distance between point $A$ and C).

\section{Materials and Methods}

The present study analyzed a CT scan of a human mandible provided by the University Hospital of Bonn, Germany, in order to evaluate two mandibular geometrical parameters (the area of the ramus largest section slice and the distance between the top point of the condyle and the minimum cancellous bone section inside the mandible). The authors were then able to validate a new and faster procedure to characterize within bone tissue (Figure 1).

Two different techniques were used to obtain the information provided by the DICOM files. The first was a 2D approach with Image-J to evaluate the interior bone characteristics. The second approach was made by Simpleware Scan IP to validate the previous method with Image-J plug-in. Simpleware Scan IP is a software that can collect and read information from DICOM files in $2 \mathrm{D}$ or 3D; however, building a 3D model could take long periods of work, depending on the user's skills, scan resolution and quality desired, which consequently preclude its wide use for clinicians and opens a window of opportunity to Image- $J$ and other open-source software.

\section{Image-J (plug-in)}

Image-J (Image analysis software v1.43 NIH, Bethesda, MD, USA, http://rsb.info.nih.gov/ij/ ) is open source software based on Java applications. This makes it extremely flexible, enabling users to read, edit, analyze, process, save and print 8-bit, 16-bit and 32-bit images in several different formats, such as TIFF, GIF, JPEG, BMP and DICOM [3,14]. Given these characteristics, and because it is within the public domain, users are allowed to modify and create new plugins for Image-J. This characteristic has generated a strong medical and academic community around new Image-J potentials, which have led to several different scripts and macros being integrated [14].

Due to these characteristics, a new Image-J plug-in called "magiKenvelop" was developed by the Research Engineer Vincent Delos from I2M, CNRS-University of Bordeaux. It allows the user to manipulate the DICOM images and creates a differentiation between cortical and cancellous bone and consequently provides the $2 \mathrm{D}$-sliced volume of the interior cancellous bone. These DICOM images were therefore loaded and the space between slices was evaluated to determine the real vertical distances in each condyle. Subsequently, in each scan one identified the top and the largest cancellous bone section slices (A and B respectively). One also evaluated the slice with the minimum cancellous bone available in the mandibular ramus $(\mathrm{C})$ (Figure 1). This procedure was performed for the right and left sides of the mandible.

Using this procedure one was able to measure:

- The area of the top of the condyle section and the minimal cancellous bone section in each condyle;

- The distance between the top of the condyle and the minimum section of cancellous bone.

In order to evaluate the accuracy/repeatability of the selections, five measurements were performed in the same patient's mandible (right ramus) for each parameter.

\section{Simpleware scanIP}

Using Simpleware scanIP, two 3D models were created based on the two extreme cases according to the quantity of cancellous bone inside the mandible (largest and smallest amounts of cancellous bone available). Simpleware scan IP allows CT data segmentation. Computed tomography images are used as a slice-by-slice source, onto which a mask is placed. This mask creates a matching between pixel location/ coordinates and bone properties which are defined by the grey-scale values. These individually sliced masks are then combined into one three-dimensional shape, which is then smoothed to minimize the occurrence of inaccurate geometry on the boundary between mask slices. Creating 3D images using this technique it is possible to create an accurate mapping of both internal and external bone surfaces [4-8].

The material properties assignment procedure is well accepted in the scientific community $[4,15,16]$. In short, it enables the material properties (density, Young's modulus and mass) to be adjusted according to the grey-scale values obtained during the CT scan. From these results, the differentiation between cancellous and cortical bone in each of our models were assumed respecting the Hounsfield units defined by Bujtar et al. [17] i.e. [600-1300] HU and [1300-1600] HU for cancellous and cortical bone respectively and respecting the software standards (Figure 2). There was a high correlation between provided by Simpleware scan IP it was possible to compare results and validate the first procedure and evaluate the accuracy of the measurements performed with Image-J.

\section{Results}

The results presented in this section intended, at first, to evaluate the repeatability of the adopted method which will promote a closer

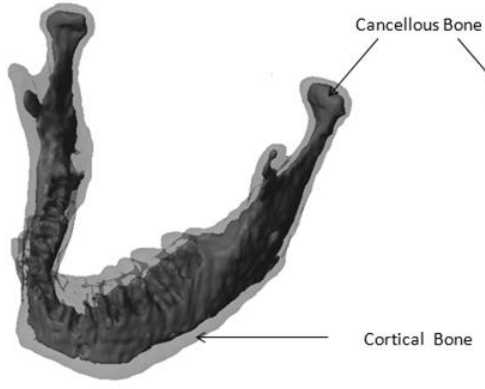

A

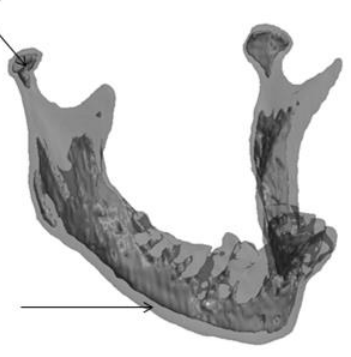

B
Figure 2: 3D model reconstruction. A: Largest amount of interior cancellous bone; B: Smallest amount of interior cancellous bone. 
Citation: Ricardo D, Vincent D, António R, Marcus T, Michel M (2015) Development of a Relevant Image Processing Method to Characterize the Distribution of Tissue within a Bone Structure. J Comput Sci Syst Biol 8: 199-202. doi:10.4172/jcsb.1000189

view over the influence of the user's skills using this method. Then, comparing with this method with commercial and accepted software, one intends to validate the accuracy of the tool performing the evaluation of bone distribution within bone structures.

\section{Repeatability of results - (standard patient)}

One observed that according to the total distance parameter (Figure $\left.1-\mathrm{X}_{1}\right)$, the repeatability test results show a range of $19-20.5 \mathrm{~mm}$ for the right condyle with a standard deviation of 0.55 (Table 1 ).

Similarly, the selected area in the right condyle shows a high repeatability rate between trials. We observed that the area results for the right condyle are between $80-85 \mathrm{~mm}^{2}$ with an average of $82 \mathrm{~mm}^{2}$. We therefore recorded a maximum error of $3 \%$ for the right condyle.

\section{Validation procedure}

To validate the first procedure one built two $3 \mathrm{D}$ models and compared the cancellous bone quantity inside the condyle as previously described. In each case, the distance between the condyle top and the smallest section of cancellous bone was evaluated and compared (Figure $3)$. There was a high correlation between Image-J and ScanIP results $\left(\mathrm{R}^{2}=0.99\right)$. This was sufficient evidence to validate the first procedure.

\section{Discussion}

The purpose of the study is to validate a new computational method to characterize the distribution of bone tissues, an essential requirement in the surgical field. Nowadays there are several technical solutions available on the market. However, the ones which offer the best results are usually associated with research and they are not considered as a surgical tool to help surgeons prepare their surgical procedures since it takes a long time to fully understand their processing methodologies and to create the models.

The main advantage of using commercial tools such as Simpleware Scan IP or similar, lies in the fact that it is possible to build a completely functional 3D model based on 2D images and export it to further numerical simulations. Despite this great advantage, there are some details that could prevent its medical use. Additionally, the procedure used to build a 3D model based on CT scans can take considerable time depending on the user's skills and the accuracy desired.

In medical practice there is always an urgency to provide a quick answer to the patient's problems. The method proposed in this study could therefore have an advantage over other complex methods, since it is faster and easier. Furthermore, although not discussed in this work, Image-J plug-in here developed can also provide a faster 3D model reconstruction based on scan images which takes just a few seconds. However, unlike other commercial solutions, with this neither model further exportation nor numerical simulation are possible as it provides just a simple 3D model that surgeons can only use to evaluate bone characteristics.

Using the parameters described in Figures 1A-1C, we evaluated the accuracy of the proposed tool and observed that the results based on the repeatability tests were very satisfactory. The five repetitions to evaluate the influence of the user's skills in selecting the section area $\mathbf{B}$ and the total distance - $\mathbf{X}_{1}$ showed that the selection did not change significantly (more than 5\%) in each trial, which represents a small influence on the part of user's expertise. Having similar results with an insignificant influence of the user's skills allows then to look for further results.

As previously stated, building 3D models using CT scan images is a well-known procedure in biomechanical research that guarantees a very satisfactory approximation to the real subject. To validate the first method described in this study (Image-J), we used a commercial solution (ScanIP. When comparing the results of these two tools we observed that the differences remained at $3 \%$ according to the parameter selected to perform the validation. This comparison points out the feasibility of the method and its potential use as a useful medical tool to evaluate bone structures and prepare a medical intervention in a faster way which permits to fill a gap according to other expensive commercial solutions.

\begin{tabular}{|l|c|c|c|c|c|c|}
\hline Essay & 1 & 2 & 3 & 4 & 5 & Average \\
\hline Distance between A and C $(\mathrm{mm})$ & 20 & 20.5 & 20 & 20 & 19 \\
\hline Area of the main section $\left(\mathrm{mm}^{2}\right)$ & 84.6 & 85.1 & 80.1 & 81.9 & 0.55 \\
\hline
\end{tabular}

Table 1: Repeatability results for the right condyle.
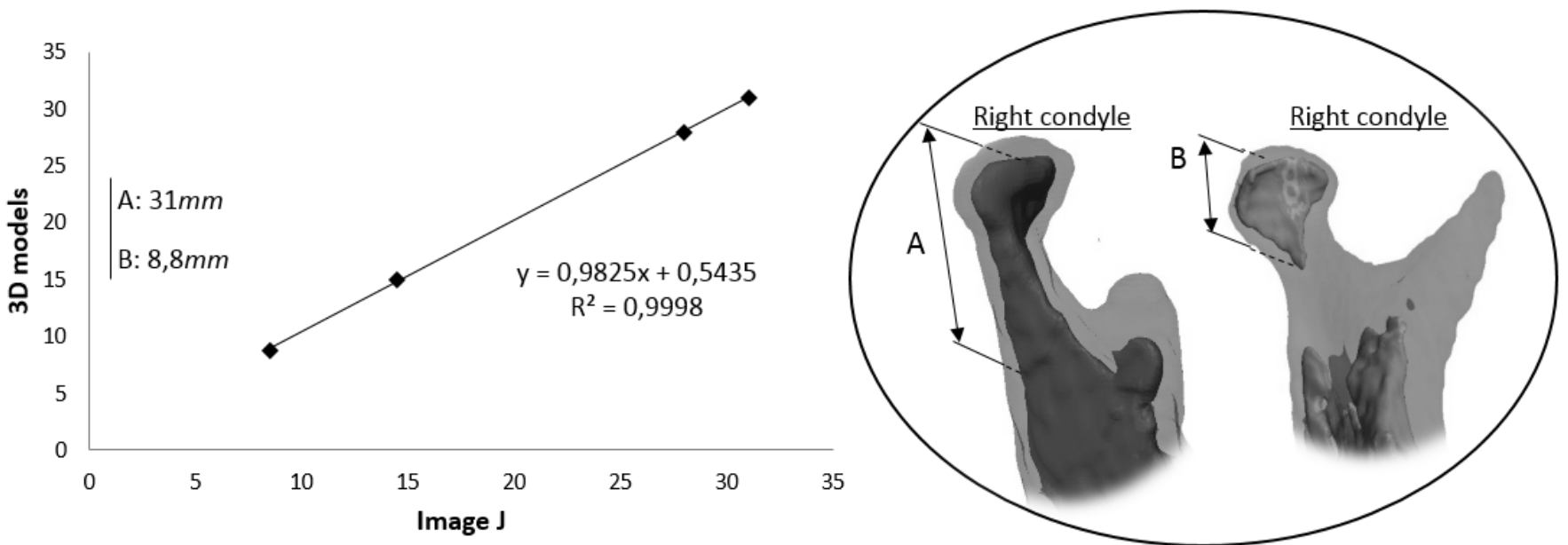

Figure 3: Protocol validation. Left: Correlation between Image-J results and 3D models; Right: 3D model measurements. 
Citation: Ricardo D, Vincent D, António R, Marcus T, Michel M (2015) Development of a Relevant Image Processing Method to Characterize the Distribution of Tissue within a Bone Structure. J Comput Sci Syst Biol 8: 199-202. doi:10.4172/jcsb.1000189

\section{Conclusion}

Processing tools can be used in different areas of academic research and medical fields as a way to predict and prepare a surgical intervention. In this study, the authors intended to validate the use of a new tool to characterize the distribution of tissues within a bone structure. Two different parameters have been compared using this software and the developed plug-in. It is now also possible to evaluate different mandible characteristics, such as cancellous bone dimensions and geometry. Comparing with other commercial tools, for example Simpleware Scan IP (used in this study), the presented method looks faster, simpler to use and having the same efficiency to produce the results which could be explored by surgeons to prepare their surgical interventions in different areas, as for example in maxillofacial surgery, knee or shoulder arthroplasty, etc. To conclude, this study promotes a closer idea to a new method to characterize the interior bone tissues which can be explored as a surgical tool in everyday surgical operations.

\section{Acknowledgement}

The authors gratefully acknowledge "Aquitaine Science Transfert" (Société d'Accélération du Transfert de Technologies).

\section{References}

1. Millesi W, Prayer L, Helmer M, Gritzmann N (1990) Diagnostic imaging of tumor invasion of the mandible. Int J Oral Maxillofac Surg 19: 294-298.

2. Shelley M, Brunton P, Horner K (2011) Subjective image quality assessment of cross sectional imaging methods for the symphyseal region of the mandible prior to dental implant placement. J Dent 39: 764-770.

3. Lind R (2012) Open source software for image processing and analysis: picture this with Image J. Open Source Softw Life Sci Res 5: 131-49.

4. Emerson NJ, Carré MJ, Reilly GC, Offiah AC (2011) Geometrically accurate 3D FE models from medical scans created to analyse the causes of sports injuries. Procedia Eng 13: 422-427.

5. Nagasao T, Aramaki-Hattori N, Shimizu Y, Yoshitatsu S, Takano N, et al. (2013) Transformation of keloids is determined by stress occurrence patterns on perikeloid regions in response to body movement. Med Hypotheses 81: 136-141.
6. Harrison GJ, How TV, Poole RJ, Brennan JA, Naik JB, et al. (2014) Closure technique after carotid endarterectomy influences local hemodynamics. J Vasc Surg 60: 418-427.

7. Majumber S, Roychowdhury A, Pal S (2007) Simulation of hip fracture in sideways fall using a 3D finite element model of pelvis-femur-soft tissue complex with simplified representation of whole body. Med Eng Phys 29: 11671178.

8. Qiu TX, Teo EC, Yan YB, Lei W (2011) Finite element modeling of a 3D coupled foot-boot model. Med Eng Phys 33: 1228-1233.

9. Lin MH, Mau LP, Cochran DL, Shieh YS, Huang PH, et al. (2014) Risk assessment of inferior alveolar nerve injury for immediate implant placement in the posterior mandible: a virtual implant placement study. J Dent 42: 263-270.

10. Koslin MG, Indresano AT, Mercuri LG (2012) Temporomandibular joint surgery. J Oral Maxillofac Surg 70: e204-231.

11. Doube M, Klosowski MM, Arganda-Carreras I, Cordelières FP, Dougherty RP et al. (2010) Bone J: Free and extensible bone image analysis in ImageJ. Bone 47: 1076-1079.

12. Torres-Espín A, Santos D, González-Pérez F, del Valle J, Navarro X2 (2014) Neurite-J: an image-J plug-in for axonal growth analysis in organotypic cultures. J Neurosci Methods 236: 26-39.

13. Xiang K, Zhu XL, Wang CX, Li BN (2013) MREJ: MRE elasticity reconstruction on ImageJ. Comput Biol Med 43: 847-852.

14. Udalagama $C$, Chen $X$, Bettiol AA, Watt $F$ (2013) An ion beam analysis software based on ImageJ. Nucl Instruments Methods Phys Res Sect B Beam Interact with Mater Atoms 306: 59-63.

15. Taddei F, Pancanti A, Viceconti M (2004) An improved method for the automatic mapping of computed tomography numbers onto finite element models. Med Eng Phys 26: 61-69.

16. Helgason B, Perilli E, Schileo E, Taddei F, Brynjólfsson S, et al. (2008) Mathematical relationships between bone density and mechanical properties: a literature review. Clin Biomech (Bristol, Avon) 23: 135-146.

17. Bujtár P, Sándor GKB, Bojtos A, Szucs A, Barabás J (2010) Finite element analysis of the human mandible at 3 different stages of life. Oral Surg Oral Med Oral Pathol Oral Radiol Endod 110: 301-309. 